Pacific Journal of Mathematics

BARE SPACES AND HYPERSPACE 


\title{
BAIRE SPACES AND HYPERSPACES
}

\author{
Robert A. McCoy
}

This paper examines the question as to when the hyperspace of a Baire space is a Baire space, and related questions. An answer is given in terms of a certain product space's being a Baire space.

A hyperspace of a space $X$ is the space of closed subsets of $X$ under a natural topology. In this paper we investigate what happens to Baire spaces in the formation of hyperspaces. The first section is devoted to a discussion of the basic concepts. In the second section some characterizations of Baire spaces are given which will be useful while working with hyperspaces, the study of which occurs in the third section. In particular, we shall be primarily concerned with two basic questions. If $X$ is a Baire space, when is the hyperspace of $X$ a Baire space? If the hyperspace of $X$ is a Baire space, when is $X$ a Baire space? We also look briefly at Baire spaces in the strong sense and pseudo-complete spaces.

1. Basic definitions and properties. A Baire space is a space in which every countable intersection of dense open subsets is dense. It can also be defined as a space such that every nonempty open subspace is of second category. The usual definition of a space of first category is one which can be written as a countable union of nowhere dense subsets (i.e., subsets whose closures have no interior points). A space is of second category then if it is not of first category. Also second category spaces can be characterized as spaces in which every countable intersection of dense open subsets is nonempty.

We now list a few of the properties which the Baire space concept enjoys. Of course the Baire Category Theorem gives a sufficient condition for a space to be a Baire space. That is, every complete metric space is a Baire space. Also every locally compact Hausdorff space is a Baire space. Clearly every nonempty open subspace of a Baire space is a Baire space. In fact a space is a Baire space if and only if every point has a neighborhood which is a Baire space. A useful property of Baire spaces is that every space which contains a dense Baire subspace is a Baire space.

The question as to which products of Baire spaces are Baire spaces has been a difficult one. There have been very few different examples 
discovered of Baire spaces whose product with itself is not a Baire space (see [9] and [6]). In $\$ 3$, we shall be interested in having $X^{\omega}$ be a Baire space. This then will be the case for most known Baire spaces $X$. In fact it will always be true that $X^{\omega}$ is a Baire space if $X$ is a Baire space having a countable base. On the otherhand if $X^{\omega}$ is a Baire space, then $X$ will always be a Baire space, since an open continuous image of a Baire space is a Baire space. A thorough investigation of the properties of Baire spaces can be found in [5].

When working with hyperspaces, we shall use the notation and terminology in [7]. Briefly, if $X$ is a topological space with topology $\mathscr{T}, 2^{X}$ denotes the set of all nonempty closed subsets of $X$ and $\mathscr{C}(X)$ denotes the set of all nonempty compact subsets of $X$. If $U_{1}, \cdots, U_{n}$ are subsets of $X$, then

$$
\begin{gathered}
\left\langle U_{1}, \cdots, U_{n}\right\rangle=\left\{A \in 2^{X} \mid A \subset \bigcup_{i=1}^{n} U_{i}\right. \text { and } \\
\left.A \cap U_{i} \neq \varnothing \text { for each } i=1, \cdots, n\right\} .
\end{gathered}
$$

The finite topology (or Vietoris topology) on $2^{x}$, denoted by $2^{g}$, is the topology generated by the base

$$
\mathscr{B}^{X}=\left\{\left\langle U_{1}, \cdots, U_{n}\right\rangle \mid U_{i} \in \mathscr{T}, i=1, \cdots, n\right\}
$$

The topology $\mathscr{T}^{c}$ on $\mathscr{C}(X)$ is the topology generated by the base $\mathscr{B}^{c}=\left\{\left\langle U_{1}, \cdots, U_{n}\right\rangle \cap \mathscr{C}(X) \mid U_{i} \in \mathscr{T}, i=1, \cdots, n\right\} . \quad$ It is easy to see that $\mathscr{C}(X)$ is a dense subspace of $2^{X}$ for $T_{1}$-spaces $X$.

If $X$ is a $T_{1}$-space, then the natural map from $X$ into $2^{X}$ which takes each $x$ onto the element $\{x\}$ of $2^{X}$ is a closed embedding. Also if $X$ is a $T_{1}$-space, so is $2^{X}$. An investigation of the properties of hyperspaces can be found in [7]. Two such very basic properties which we shall implicityly use are the following.

$\left\langle U_{1}, \cdots, U_{n}\right\rangle \subset\left\langle V_{1}, \cdots, V_{m}\right\rangle$ if and only if $\cup_{i=1}^{n} U_{i} \subset \cup_{i=1}^{n} V_{i}$ and for each $V_{i}$ there exists a $U_{j}$ such that $U_{j} \subset V_{i}$; and $\operatorname{Cl}\left(\left\langle U_{1}, \cdots, U_{n}\right\rangle\right)=\left\langle\bar{U}_{1}, \cdots, \bar{U}_{n}\right\rangle$ (where ClU and $\bar{U}$ both denote the closure of $U$ ).

We shall occasionally be concerned with quasi-regular spaces, that is, spaces such that every nonempty open set contains a closed subset with nonempty interior. It is not difficult to see that if $X$ is quasiregular, then $2^{X}$ and $\mathscr{C}(X)$ are quasi-regular. 
2. Characterizations of Baire spaces. In $\$ 3$, we shall need ways of looking at Baire spaces other than our definition. In this section, we establish the needed characterizations.

Let $X$ be an arbitrary topological space. By a pseudo-base for $X$ is meant a collection of nonempty open subsets of $X$ such that each nonempty open subset of $X$ contains a member of this collection.

Let $\mathscr{B}$ be a pseudo-base for $X$. Define

$$
\begin{aligned}
S(X, \mathscr{B}) & =\{f: \mathscr{B} \rightarrow \mathscr{B} \mid f(U) \subset U \text { for every } U \in \mathscr{B}\}, \text { and } \\
R S(X, \mathscr{B}) & =\{f: \mathscr{B} \rightarrow \mathscr{B} \mid \overline{f(U)} \subset U \text { for every } U \in \mathscr{B}\} .
\end{aligned}
$$

If $U \in \mathscr{B}$ and $f, g \in S(X, B)$ or $R S(X, \mathscr{B})$, define

$$
\begin{aligned}
& {[U, f, g]_{1}=g(U), \text { and for } i>1,} \\
& {[U, f, g]_{i}= \begin{cases}f\left([U, f, g]_{i-1}\right), & \text { if } i \text { is even, } \\
g\left([U, f, g]_{i-1}\right), & \text { if } i \text { is odd. }\end{cases} }
\end{aligned}
$$

The following theorem can be found in [5], and similar theorems can be found in [6] and [8].

THEOREM 2.1. The following are equivalent.

(i) $X$ is a Baire space.

(ii) There exists a pseudo-base $\mathscr{B}$ for $X$ such that for every $U \in \mathscr{B}$ and $f \in S(X, \mathscr{B})$, there exists a $g \in S(X, \mathscr{B})$ such that

$$
\bigcap_{i=1}^{\infty}[U, f, g]_{i} \neq \varnothing \text {. }
$$

(iii) For every pseudo-base $\mathscr{B}$ for $X$ and every $U \in \mathscr{B}$ and $f \in$ $S(X, \mathscr{B})$, there exists a $g \in S(X, \mathscr{B})$ such that $\cap_{i=1}^{\infty}[U, f, g]_{i} \neq \varnothing$.

There is an analogous result for spaces of second category.

THEOREM 2.2. The following are equivalent.

(i) $X$ is of second category.

(ii) There exists a pseudo-base $\mathscr{B}$ for $X$ such that for every $f \in S(X, \mathscr{B})$, there exists a $U \in \mathscr{B}$ and $g \in S(X, \mathscr{B})$ such that $\cap_{i=1}^{\infty}[U, f, g]_{i} \neq \varnothing$.

(iii) For every pseudo-base $\mathscr{B}$ for $X$ and every $f \in S(X, \mathscr{B})$, there exists $U \in \mathscr{B}$ and $g \in S(X, \mathscr{B})$ such that $\cap_{i=1}^{\infty}[U, f, g]_{i} \neq \varnothing$.

The characterization which we actually use in the next section is given in the following theorem. 
THEORem 2.3. Let $X$ be a quasi-regular space, and let $\mathscr{B}$ be a pseudo-base for $X$. Then the following are equivalent.

(i) $X$ is a Baire space.

(ii) For every $U \in B$ and $f \in S(X, \mathscr{B})$, there exists $g \in R S(X, \mathscr{B})$ such that $\cap_{i=1}^{\infty}[U, f, g]_{i} \neq \varnothing$.

(iii) For every $U \in \mathscr{B}$ and $f \in R S(X, \mathscr{B})$, there exists $g \in$ $R S(X, \mathscr{B})$ such that $\cap_{i=1}^{\infty}[U, f, g]_{i} \neq \varnothing$.

(iv) For every $U \in \mathscr{B}$ and $f \in R S(X, \mathscr{B})$, there exists $g \in S(X, \mathscr{B})$ such that $\cap_{i=1}^{\infty}[U, f, g]_{i} \neq \varnothing$.

Proof. For every $U \in \mathscr{B}, f, g \in S(X, \mathscr{B})$ with $f(U) \neq U$ and $\overline{g(U)} \subset U$, define $\hat{f}, \hat{g} \in R S(X, \mathscr{B})$ as follows. Since $X$ is quasi-regular, for every $V \in \mathscr{B}$, there exists $\hat{f}(V) \in B$ such that $\overline{f(V)} \subset f(V)$. If $V=f\left([U, \hat{f}, g]_{2 n-1}\right)$ for some $n=1,2, \cdots$, let $\hat{g}(V)=[U, \hat{f}, g]_{2 n+1}$; if $V=$ $U$, let $\hat{g}(V)=g(V)$; and otherwise let $\hat{g}(V)$ be an element of $\mathscr{B}$ such that $\hat{g}(V) \subset V$. Now observe that $[U, f, \hat{g}]_{1}=\hat{g}(U)=g(U)=$ $[U, \hat{f}, g]_{1}$. Suppose that $[U, f, \hat{g}]_{2 k-1}=[U, \hat{f}, g]_{2 k-1}$ for every $k=$ $1, \cdots, n$. Then $[U, f, \hat{g}]_{2 n+1}=\hat{g}\left(f\left([U, f, \hat{g}]_{2 n-1}\right)\right)=\hat{g}\left(f\left([U, \hat{f}, g]_{2 n-1}\right)\right)=$ $[U, \hat{f}, g]_{2 n+1}$. Therefore by induction $[U, f, \hat{g}]_{2 n-1}=[U, \hat{f}, g]_{2 n-1}$ for every $n$.

To see that (i) imples (ii), suppose that (ii) does not hold. Then there exists $U \in \mathscr{B}$ and $f \in S(X, \mathscr{B})$ such that for every $g \in R S(X, \mathscr{B})$, $\cap_{i=1}^{\infty}[U, f, g]_{i}=\varnothing$. Let $g$ be an arbitrary element of $S(X, \mathscr{B})$. We may assume without loss of generality that $f(U) \neq U$ and $\overline{g(U)} C$ $U$. Now $\cap_{i=1}^{\infty}[U, \hat{f}, g]_{i}=\cap_{i=1}^{\infty}[U, f, \hat{g}]_{i}=\varnothing$, so that $X$ cannot be a Baire space by Theorem 2.1.

Clearly (ii) implies (iii) and (iii) implies (iv), so that it remains to show that (iv) implies (i). Suppose $X$ is not a Baire space. Then there exists $U \in \mathscr{B}$ and $f \in S(X, \mathscr{B})$ such that for every $g \in S(X, \mathscr{B})$, $\cap_{i=1}^{\infty}[U, f, g]_{i}=\varnothing$. Let $g$ be an arbitrary element of $S(X, \mathscr{B})$. Again we may assume without loss of generality that $f(U) \neq U$ and $\overline{g(U)} \subset$ $U$. Then $\cap_{i=1}^{\infty}[U, \hat{f}, g]_{i}=\cap_{i=1}^{\infty}[U, f, \hat{g}]_{i}=\varnothing$, which establishes the negation of statement (iv) by Theorem 2.1 again.

Just as Theorem 2.1 has Theorem 2.2 as an analog for spaces of second category, Theorem 2.3 has its analog for spaces of second category, which we shall not state since it can easily be induced.

3. Applications to hyperspaces. First we establish that for a $T_{1}$-space $X$, if the hyperspace of $X$ is a Baire space, then $X$ must be a Baire space. Since the following two theorems have essentially the same proofs, we only give the proof for the second theorem. 
THEOREM 3.1. If $\mathscr{C}(X)$ is a Baire space (space of second category, respectively), then $X$ is a Baire space (space of second category, respectively).

THEOREM 3.2. If $X$ is a $T_{1}$-space and $2^{X}$ is a Baire space (space of second category, respectively), then $X$ is a Baire space (space of second category, respectively).

Proof. Suppose that $X$ is not a Baire space. Then there exists an open $U$ in $X$ and a sequence $\left\{U_{i}\right\}$ of dense open subsets of $X$ such that $U \cap\left(\cap_{i=1}^{\infty} U_{i}\right)=\varnothing$. Let $G=\langle U\rangle$, and for each $i$, let $G_{i}=\left\langle U_{i}\right\rangle$. To see that each $G_{i}$ is dense in $2^{x}$, let $\left\langle V_{1}, \cdots, V_{n}\right\rangle$ be an element of $\mathscr{B}^{x}$. Now each $V_{j} \cap U_{i} \neq \varnothing$ for $j=1, \cdots, n$. So let $x_{j} \in V_{j} \cap U_{i}$ for each such $j$, and let $A=\left\{x_{1}, \cdots, x_{n}\right\}$. Since $X$ is a $T_{1}$-space, $A \in$ $2^{X}$. Also $A$ is easily seen to be in $G_{i} \cap\left\langle V_{1}, \cdots, V_{n}\right\rangle$, so that each $G_{i}$ is dense in $2^{X}$. Finally, it is clear that $G \cap\left(\cap_{i=1}^{\infty} G_{i}\right)=\varnothing$, so that $2^{X}$ could not be a Baire space.

Corollary 3.3. If $X$ is a first category space, then so is $\mathscr{C}(X)$. If in addition $X$ is a $T_{1}$-space, then $2^{X}$ is a first category $T_{1}$-space.

The $T_{1}$-space hypothesis in Theorem 3.2 cannot be omitted since the space of natural numbers, $N$, with the topology consisting of right rays is a space of first category, while $2^{N}$ has the indiscrete topology, so is a Baire space. Note that this is also an example of a space $X$ such that $2^{X}$ is quasi-regular, but $X$ is not quasi-regular.

Before examining the converses of Theorem 3.1 and 3.2, we wish to introduce a new space. Let $X^{\omega}$ denote the Cartesian product of a countably infinite number of copies of $X$. Instead of putting the usual product topology on $X^{\omega}$, we shall need to consider a topology $\mathscr{T}^{*}$ on $X^{\omega}$ which lies strictly between the product topology and the box topology. If $U_{1}, \cdots, U_{n}$ are subsets of $X$, let $\Pi\left(U_{1}, \cdots, U_{n}\right)$ denote the subset $\left(\prod_{i=1}^{n} U_{i}\right) \times\left(\prod_{i=n+1}^{\infty}\left(\cup_{j=1}^{n} U_{j}\right)\right)$ of $X^{\omega}$. Let

$$
\mathscr{B}^{*}=\left\{\Pi\left(U_{1}, \cdots, U_{n}\right) \mid U_{i} \text { is open in } X, i=1, \cdots, n\right\}
$$

Finally, let $\mathscr{T}^{*}$ be the topology on $X^{\omega}$ generated by $\mathscr{B}^{*}$.

Lemma 3.4. The family $\mathscr{B}^{*}$ is a base for $\left(X^{\omega}, \mathscr{T}^{*}\right)$.

Proof. Let $U=\Pi\left(U_{1}, \cdots, U_{n}\right)$ and $V=\Pi\left(V_{1}, \cdots, V_{m}\right)$ be arbitrary members of $\mathscr{B}^{*}$. We may suppose that $n \geqq m$. Then 


$$
\begin{gathered}
U \cap V=\Pi\left(U_{1} \cap V_{1}, \cdots, U_{m} \cap V_{m}, U_{m+1} \cap\left(\bigcup_{i=1}^{m} V_{i}\right), \cdots, U_{n}\right. \\
\left.\cap\left(\bigcup_{i=1}^{m} V_{i}\right),\left(\bigcup_{i=1}^{n} U_{i}\right) \cap\left(\bigcup_{i=1}^{m} V_{i}\right)\right),
\end{gathered}
$$

which is a member of $\mathscr{B}^{*}$.

Lemma 3.4 now gives us the following fact.

Lemma 3.5. Each projection map on $\left(X^{\omega}, \mathscr{T}^{*}\right)$ is continuous and open.

It is easy to see that open continuous functions preserve Baire spaces and spaces of second category (see [4]). The next lemma then follows from Lemma 3.5.

LEMma 3.6. If $\left(X^{\omega}, \mathscr{T}^{*}\right)$ is a Baire space (space of second cate. gory, respectively), then $X$ is a Baire space (space of second category, respectively).

A modification of the proofs of 2.5 and 2.6 in [9] establishes the following fact.

Lемма 3.7. If $X$ is a Baire space (space of second category, respectively) having a countable pseudo-base, then $\left(X^{\omega}, \mathscr{J}^{*}\right)$ is a Baire space (pace of second category, respectively).

We now introduce some notation which will be used in the proof of the next two theorems. We shall be working with the three spaces $\left(X^{\omega}, \mathscr{T}^{*}\right),\left(2^{X}, 2^{\mathscr{T}}\right)$, and $\left(\mathscr{C}(X), \mathscr{T}^{c}\right)$ having bases $\mathscr{B}^{*}, \mathscr{B}^{X}$, and $\mathscr{B}^{c}$, respectivley. If $U=\Pi\left(U_{1}, \cdots, U_{n}\right) \in \mathscr{B}$, then define $U^{X}=$ $\left\langle U_{1}, \cdots, U_{n}\right\rangle$, which is an element of $\mathscr{B}^{X}$, and define $U^{c}=$ $\left\langle U_{1}, \cdots, U_{n}\right\rangle \cap \mathscr{C}(X)$, which is an element of $\mathscr{B}^{c}$. On the other hand if $U=\left\langle U_{1}, \cdots, U_{n}\right\rangle \in \mathscr{B}^{X}$ or $U=\left\langle U_{1}, \cdots, U_{n}\right\rangle \cap \mathscr{C}(X) \in \mathscr{B}^{c}$, define $U^{*}=$ $\Pi\left(U_{1}, \cdots, U_{n}\right)$, which is an element of $\mathscr{B}^{*}$. Also for each $f \in$ $R S\left(X^{\omega}, \mathscr{B}^{*}\right)$, define $f^{X} \in R S\left(2^{X}, \mathscr{B}^{X}\right)$ and $f^{c} \in R S\left(\mathscr{C}(X), \mathscr{B}^{c}\right)$ as follows. If $U=\left\langle U_{1}, \cdots, U_{n}\right\rangle$ is an arbitrary element of $\mathscr{B}^{X}$, define $f^{X}(U)=\left(f\left(U^{*}\right)\right)^{X}$, and if $U=\left\langle U_{1}, \cdots, U_{n}\right\rangle \cap \mathscr{C}(X)$ is an arbitrary element of $\mathscr{B}^{c}$, define $f^{c}(U)=\left(f\left(U^{*}\right)\right)^{c}$. Finally, for each $f \in R S\left(2^{X}, \mathscr{B}^{X}\right)$ or $f \in R S\left(\mathscr{C}(X), \mathscr{B}^{c}\right)$, define $f^{*} \in R S\left(X^{\omega}, \mathscr{B}^{*}\right)$ as follows. Let $f \in$ $R S\left(2^{X}, \mathscr{B}^{X}\right)$. If $U=\left\langle U_{1}, \cdots, U_{n}\right\rangle \in \mathscr{B}^{X}$, then we may assume that $f(U)$ is written as $\left\langle V_{1}, \cdots, V_{m}\right\rangle$, where $m \geqq n$ and for each $i=1, \cdots, n$, $\bar{V}_{i} \subset U_{i}$. Now if $U=\Pi\left(U_{1}, \cdots, U_{n}\right)$ is an arbitrary element of $\mathscr{B}^{*}$, then define $f^{*}(U)=\left(f\left(U^{X}\right)\right)^{*}$. A similar definition is to be given for $f^{*}$ if $f \in R S\left(\mathscr{C}(X), \mathscr{B}^{c}\right)$. 
TheOREM 3.8. If $X$ is quasi-regular and $\left(X^{\omega}, \mathscr{T}^{*}\right)$ is a Baire space (space of second category, respectively), then $2^{X}$ is a Baire space (space of second category, respectively).

Proof. Let $U \in \mathscr{B}^{X}$ and let $f \in R S\left(2^{x}, \mathscr{B}^{x}\right)$. Since $\left(X^{\omega}, \mathscr{T}^{*}\right)$ is a Baire space, by Theorem 2.3 , there exists $g \in R S\left(X^{\omega}, \mathscr{B}^{*}\right)$ such that $\cap_{i=1}^{\infty}\left[U^{*}, f^{*}, g\right]_{i}$ contains some element $\left(x_{i}\right)$ of $X^{\omega}$. Now $\left[U, f, g^{X}\right]_{2}=$ $f\left(g^{X}(U)\right)=f\left(\left(g\left(U^{*}\right)\right)^{X}\right), \quad$ and $\left(f\left(\left(g\left(U^{*}\right)\right)^{X}\right)\right)^{*}$. Also for each $n>1$,

$$
\begin{aligned}
{\left[U, f, g^{X}\right]_{2 n} } & =f\left(g^{X}\left(\left[U, f, g^{X}\right]_{2 n-2}\right)\right) \\
& =f\left(\left(g\left(\left[U, f, g^{X}\right]_{2 n-2}^{*}\right)\right)^{X}\right), \quad \text { and } \\
{\left[U^{*}, f^{*}, g\right]_{2 n} } & =f^{*}\left(g\left(\left[U^{*}, f^{*}, g\right]_{2 n-2}\right)\right) \\
& =\left(f\left(\left(g\left(\left[U^{*}, f^{*}, g\right]_{2 n-2}\right)\right)^{X}\right)\right)^{*} .
\end{aligned}
$$

So that by induction, $\left[U^{*}, f^{*}, g\right]_{2 n}=\left(\left[U, f, g^{x}\right]_{2 n}\right)^{*}$ for every $n$. Therefore since $\left(x_{i}\right) \in\left[U^{*}, f^{*}, g\right]_{2 n}$ for every $n$, then $\left\{\overline{x_{i}}\right\} \in$ $\left[U, f, g^{x}\right]_{2 n}$ for every $n$. Thus $\cap_{i=1}^{\infty}\left[U, f, g^{x}\right]_{i} \neq \varnothing$, so that $2^{X}$ must be a Baire space by Theorem 2.3 again.

COROllary 3.9. If $X$ is a quasi-regular Baire space (space of second category, respectively) having a countable pseudo-base, then $2^{X}$ is a Baire space (space of second category, respectively).

If we consider the space $\mathscr{C}(X)$ instead of $2^{X}$, then we obtain the converse of Theorem 3.8 .

THEOREM 3.10. If $X$ is quasi-regular and $\mathscr{C}(X)$ is a Baire space (space of second category, respectively), then $\left(X^{\omega}, \mathscr{T}^{*}\right)$ is a Baire space (space of second category, respectively).

Proof. Let $U \in \mathscr{B}^{*}$ and let $f \in R S\left(X^{\omega}, \mathscr{B}^{*}\right)$. Since $\mathscr{C}(X)$ is a Baire space, by Theorem 2.3, there exists a $g \in R S\left(\mathscr{C}(X), \mathscr{B}^{c}\right)$ such that $\cap_{i=1}^{\infty}\left[U^{c}, f^{c}, g\right]_{i}$ contains some element $A$ of $\mathscr{C}(X)$. Now $\left[U, f, g^{*}\right]_{2}=f\left(g^{*}(U)\right)=f\left(\left(g\left(U^{c}\right)\right)^{*}\right), \quad$ and $\quad\left[U^{c}, f^{c}, g\right]_{2}=f^{c}\left(g\left(U^{c}\right)\right)=$ $\left.f\left(\left(g\left(U^{c}\right)\right)^{*}\right)\right)^{c}$. Also for each $n>1$,

$$
\begin{aligned}
{\left[U, f, g^{*}\right]_{2 n} } & =f\left(g^{*}\left(\left[U, f, g^{*}\right]_{2 n-2}\right)\right) \\
& =f\left(\left(g\left(\left[U, f, g^{*}\right]_{2 n-2}^{c}\right)\right)^{*}\right), \text { and } \\
{\left[U^{c}, f^{c}, g\right]_{2 n} } & =f^{c}\left(g\left(\left[U^{c}, f^{c}, g\right]_{2 n-2}\right)\right) \\
& =\left(f\left(\left(g\left(\left[U^{c}, f^{c}, g\right]_{2 n-2}\right)\right)^{*}\right)\right)^{c} .
\end{aligned}
$$


So that by induction, $\left[U^{c}, f^{c}, g\right]_{2 n}=\left(\left[U, f, g^{*}\right]_{2 n}\right)^{c}$ for every $n$. Suppose that for each $n,\left[U^{c}, f^{c}, g\right]_{2 n}=\left\langle U_{1}^{n}, \cdots, U_{m(n)}^{n}\right\rangle \cap \mathscr{C}(X)$. Then each $\left[U, f, g^{*}\right]_{2 n}=\left(U_{1}^{n}, \cdots, U_{m(n)}^{n}\right)$, so that $m(1) \leqq m(2) \leqq \cdots$, and for each $i=1, \cdots, m(n), U_{i}^{n+1} \subset U_{i}^{n}$.

Let $i$ be a fixed positive integer. If there exists an $n$ such that $i \leqq m(n)$, let $n(i)$ be the smallest such $n$. In this case, for each $j=n(i), n(i)+1, \cdots$, let $a_{i}^{j} \in A \cap U_{i}^{i}$. Otherwise, for each $j=$ $1,2, \cdots$, let $a_{i}^{i} \in A \cap\left(\cup_{k=1}^{m(j)} U_{k}^{j}\right)$. In either case, since $A$ is compact, $\left\{a_{i}^{j} \mid j=1,2, \cdots\right\}$ has a cluster point, say $a_{i}$. Now letting $i$ vary, this defines the point $\left(a_{i}\right) \in X^{\omega}$. It can be seen that $\left(a_{i}\right) \in \cap_{i=1}^{\infty}\left[U, f, g^{*}\right]_{i}$, so that $\left(X^{\omega}, \mathscr{T}^{*}\right)$ must be a Baire space by Theorem 2.3 again.

We might observe that when $X$ is a quasi-regular space, Theorem 3.1 now follows from Theorem 3.10 and Lemma 3.6.

Using Theorem 3.10, we can now obtain an example of a metric Baire space $K$ such that $\mathscr{C}(K)$ is not a Baire space. In [9], Oxtoby gave an example of a Baire space whose square is not a Baire space. This space unfortunately needs the continuum hypothesis in its construction. Later in [6], Krom constructed a new space $K$ from Oxtoby's example which is a metric Baire space whose square is not a Baire space. Now if $X^{2}$ is not a Baire space, it is easy to see by a modification of Lemma 3.6 that $\left(X^{\omega}, \mathscr{T}^{*}\right)$ is not a Baire space. Thus by Theorem $3.10, \mathscr{C}(K)$ is not a Baire space.

It would be of interest to know whether $2^{K}$ is a Baire space, where $K$ is Krom's example discussed above. One is tempted to try to answer this by investigating the relationship between $2^{\mathrm{X}}$ and $\mathscr{C}(X)$ in terms of being a Baire space. First, since any extension of a Baire space is a Baire space, we immediately get the following.

\section{Theorem 3.11. If $\mathscr{C}(X)$ is a Baire space, then so is $2^{X}$.}

On the other hand, Aarts and Lutzer in [1] gave a test to determine when a dense subspace of a Baire space is a Baire space. A modification of this theorem (see [5]) is that if $X$ is a dense subspace of the Baire space $Y$, then $X$ is a Baire space if and only if every somewhere dense $G_{\delta}$-subset of $Y$ intersects $X$ (a set being somewhere dense if its closure has nonempty interior). Therefore, as partial converse of Theorem 3.11 , we have the following.

THEOREM 3.12. If $2^{X}$ is a Baire space and every somewhere dense $G_{\delta}$-subset of $2^{X}$ intersects $\mathscr{C}(X)$, then $\mathscr{C}(X)$ is a Baire space.

However, the full converse of Theorem 3.9 is false, as we shall now extablish. Let $P$ be a dense Baire subspace of the irrationals with the 
usual metric topology having the property that every compact subset has an isolated point (see for example [1], example 2.4; or [5], Theorem 2.6). Let $\mathscr{I}$ be the set of all intervals in $P$ having rational end points and diameter less than one. For each pairwise disjoint collection $\left\{I_{1}, \cdots, I_{k}\right\}$ of elements of $\mathscr{I}$ and for each natural number $n$, let

$$
G_{n}\left(I_{1}, \cdots, I_{k}\right)=\left\langle I_{1}^{1}, \cdots, I_{1}^{n}, I_{2}^{1}, \cdots, I_{2}^{n}, \cdots, I_{k}^{1}, \cdots, I_{k}^{n}\right\rangle
$$

where for each $i=1, \cdots, k$, the $I_{i}^{j}$ 's are pairwise disjoint intervals such that $I_{i}=\cup_{j=1}^{n} I_{i}^{j}$ and $\operatorname{diam} I_{i}^{j}=1 / n \operatorname{diam} I_{i}$ for each $j=1, \cdots, n$. Now for each $n$, let $G_{n}=\cup\left\{G_{n}\left(I_{1}, \cdots, I_{k}\right) \mid I_{1}, \cdots, I_{k}\right.$ are pairwise disjoint elements of $\mathscr{I}\}$, which is a dense open subset of $2^{P}$. To see that $\left(\cap_{n=1}^{\infty} G_{n}\right) \cap \mathscr{C}(P)=\varnothing$, let $A \in \mathscr{C}(P)$. Now $A$ must contain some isolated point $x$. Let $m$ be a natural number such that no other point of $A$ is within $3 / m$ of $x$. If for some $I_{1}, \cdots, I_{k} \in \mathscr{I}, A \in G_{m}\left(I_{1}, \cdots, I_{k}\right)$, then $x \in I_{i}^{j}$ for some $i=1, \cdots, k$ and $j=1, \cdots, m$. But then for some $j^{\prime}=1, \cdots, m, \quad A \cap I_{i}^{j^{\prime}}=\varnothing-$ which contradicts $A$ being in $G_{m}\left(I_{1}, \cdots, I_{k}\right)$. Therefore $\left(\cap_{n=1}^{\infty} G_{n}\right) \cap \mathscr{C}(P)=\varnothing$, so that $\mathscr{C}(P)$ must be of first category. Note also that $\cap_{n=1}^{\infty} G_{n}$ is a dense $G_{\delta}$-subset of $2^{P}$. Finally since $P$ is second countable, $2^{P}$ will be a Baire space by Corollary 3.9.

For the final two theorems, we shall be concerned with two properties closely related to Baire spaces - Baire spaces in the strong sense and pseudo-complete spaces.

A space $X$ is called a Baire space in the strong sense (or totally non-meagre space) provided that every nonempty closed subspace of $X$ is of second category. This turns out to be equivalent to the property that every nonempty closed subspace is a Baire space. Discussions of Baire spaces in the strong sense can be found in [3] and [2]. We have the following immediate theorem.

Theorem 3.13. Let $X$ be a $T_{1}$-space. If either $2^{X}$ or $\mathscr{C}(X)$ is a Baire space in the strong sense, then so is $X$.

A space $X$ is called pseudo-complete if it is quasi-regular and there exists a sequence $\left\{\mathscr{B}_{i}\right\}$ of pseudo-bases for $X$ such that if for each $i=2, \cdots, U_{i} \in \mathscr{B}_{i}$ and $\overline{U_{i+1}} \subset U_{i}$, then $\cap_{i=1}^{\infty} U_{i} \neq \varnothing$. It is not difficult to see that every pseudo-complete space is a Baire space. Properties of pseudo-complete spaces can be found in [9] and [1]. One such interesting property of pseudo-complete spaces (found in the latter reference) is that for a metric space $X, X$ is pseudo-complete if and only if it has a dense completely metrizable subspace. 
THEOREM 3.14. If $X$ is pseudo-complete, then so is $2^{X}$.

Proof. Let $\left\{\mathscr{B}_{i}\right\}$ be a sequence of pseudo-bases for $X$ such that if for each $i=1,2, \cdots, U_{i} \in \mathscr{B}_{i}$ and $\overline{U_{i+1}} \subset U_{i}$, then $\cap_{i=1}^{\infty} U_{i} \neq \varnothing$. For each $i$, let $\mathscr{B}_{i}^{X}=\left\{\left\langle U_{1}, \cdots, U_{n}\right\rangle \mid U_{1}, \cdots, U_{n} \in \mathscr{B}_{i}\right\}$, which is a pseudo-base for $2^{X}$. Now for each $i$, let $G_{i}=\left\langle U_{i}^{i}, \cdots, U_{n(i)}^{i}\right\rangle \in \mathscr{B}_{i}^{X}$ such that $\overline{G_{i+1}} \subset$ $G_{i}$. We may assume that $n(1) \leqq n(2) \leqq \cdots$, and that for each $i$, $U_{j}^{i+1} \subset U_{j}^{i}$ for $j=1, \cdots, n(i)$. Also we know for each $i$ that $U_{j=1}^{n(i+1)} \overline{U_{j}^{i+1}} \subset \cup_{j=1}^{n(i)} U_{j}^{i}$. Let $n(0)=1$. For each nonnegative integer $k$ such that $n(k)<n(k+1)$ and each integer $j$ such that $n(k)<j \leqq$ $n(k+1)$, choose $x_{j} \in \cap_{i=k+1}^{\infty} U_{j}^{i}$. Let $L=\infty$ if $\lim _{k \rightarrow \infty} n(k)$ is infinite, and let $L=1+\lim _{k \rightarrow \infty} n(k)$ if $\lim _{k \rightarrow \infty} n(k)$ is finite. Then define $A=$ $\left\{x_{j} \mid 1 \leqq j<L\right\}$. Now $A \in G_{i}$ for every $i$, so that $\bar{A} \in \overline{G_{i+1}} \subset G_{i}$ for every $i$. Therefore $\bar{A} \in \cap_{i=1}^{\infty} G_{i}$, so that $2^{x}$ is pseudo-complete.

The converse of Theorem 3.14 is easily seen to be false since the space $N$ of natural numbers with the right ray topology is not quasiregular, while $2^{x}$ has the indiscrete topology and is hence pseudocomplete.

\section{REFERENCES}

1. J. M. Aarts and D. J. Lutzer, Pseudo-completeness and the product of Baire spaces, Pacific J. Math., 48 (1973), 1-10.

2. _- The product of totally nonmeagre spaces, Proc. Amer. Math. Soc., 38 (1973), 198-200.

3. N. Bourbaki, General Topology, part 2, Addison-Wesley Publishing Company, Reading, Massachusetts, 1966.

4. Z. Frolik, Remarks concerning the invariance of Baire spaces under mappings, Czech. Math. J., 11 (1961), 381-385.

5. R. C. Haworth and R. A. McCoy, Baire spaces, (to appear).

6. M. R. Krom, Cartesian products of metric Baire spaces, Proc. Amer. Math. Soc., 42 (1973), 588-594.

7. E. Michael, Topologies on spaces of subsets, Trans. Amer. Math. Soc., 71 (1951), 152-182.

8. J. C. Oxtoby, The Banach-Mazur game and Banach Category Theorem, Contribution to the theory of games Vol. III, (Annals of Mathematics Studies number 39), Princeton University Press, Princeton, 1957.

9. _- Cartesian products of Baire spaces, Fund. Math., 49 (1961), 157-166.

Received February 28, 1974 and in revised form May 28, 1974.

VPI \& SU, BLACKSBURG. VIRGINIA 


\section{CONTENTS}

Zvi Artstein and John A. Burns, Integration of compact set-valued functions

J. A. Beachy and W. D. Blair, Rings whose faithful left ideals are cofaithful

Mark Benard, Characters and Schur indices of the unitary reflection group $[321]^{3}$

H. L. Bentley and B. J. Taylor, Wallman rings ............................. 15

E. Berman, Matrix rings over polynomial identity rings II ............... 37

Simeon M. Berman, A new characterization of characteristic functions of absolutely continuous distributions ........................ 323

Monte B. Boisen, Jr. and Philip B. Sheldon, Pre-Prüfer rings ............ 331

A. K. Boyle and K. R. Goodearl, Rings over which certain modules are injective

J. L. Brenner, R. M. Crabwell and J. Riddell, Covering theorems for finite nonabelian simple groups. $V$

H. H. Brungs, Three questions on duo rings .................................... 345

Iracema M. Bund, Birnbaum-Orlicz spaces of functions on groups ....351

John D. Elwin and Donald R. Short, Branched immersions between 2-manifolds of higher topological type

J. K. Finch, The single valued extension property on a Banach space ................................................................................. 61

J. R. Fisher, A Goldie theorem for differentiably prime rings ............ 71

Eric M. Friedlander, Extension functions for rank 2, torsion free abelian groups ...................................................................... 371

J. Froemke and R. Quackenbusch, The spectrum of an equational class of groupoids

B. J. Gardner, Radicals of supplementary semilattice sums of

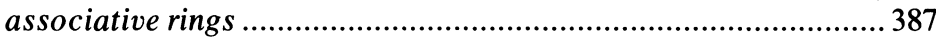

Shmuel Glasner, Relatively invariant measures ...............................393

G. R. Gordh, Jr. and Sibe Mardešić, Characterizing local connectedness in inverse limits...

S. Graf, On the existence of strong liftings in second countable topological spaces

S. Gudder and D. Strawther, Orthogonally additive and orthogonally increasing functions on vector spaces ........................................427

F. Hansen, On one-sided prime ideals .......................................... 79

D. J. Hartfiel and C. J. Maxson, A characterization of the maximal monoids and maximal groups in $\beta x$.

Robert E. Hartwig and S. Brent Morris, The universal flip matrix and the generalized faro-shuffle 


\section{Pacific Journal of Mathematics}

Vol. 58, No. 1

March, 1975

John Allen Beachy and William David Blair, Rings whose faithful left ideals are cofaithful .................................... 1

Herschel Lamar Bentley and Barbara June Taylor, Wallman rings ........ 15

Elizabeth Berman, Matrix rings over polynomial identity rings. II ...... 37

Ann K. Boyle and Kenneth R. Goodearl, Rings over which certain modules are injective ................................. 43

J. L. Brenner, Robert Myrl Cranwell and James Riddell, Covering theorems for finite nonabelian simple groups. $V \ldots \ldots \ldots \ldots \ldots \ldots \ldots \ldots \ldots$

James Kenneth Finch, The single valued extension property on a Banach

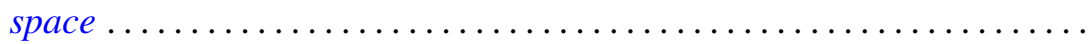

John Robert Fisher, A Goldie theorem for differentiably prime rings........ Friedhelm Hansen, On one-sided prime ideals .................... Jon Craig Helton, Product integrals and the solution of integral equations..........................................

Barry E. Johnson and James Patrick Williams, The range of a normal

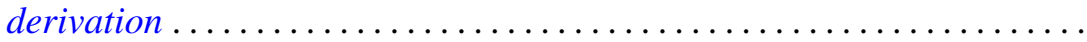

Kurt Kreith, A dynamical criterion for conjugate points ............ 123

Robert Allen McCoy, Baire spaces and hyperspaces .............. 133

John McDonald, Isometries of the disk algebra ................ 143

H. Minc, Doubly stochastic matrices with minimal permanents ......... 155

Shahbaz Noorvash, Covering the vertices of a graph by vertex-disjoint paths. ...

Theodore Windle Palmer, Jordan *-homomorphisms between reduced Banach*-algebras

Donald Steven Passman, On the semisimplicity of group rings of some

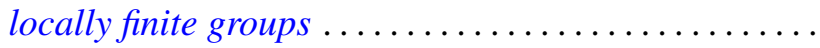

Mario Petrich, Varieties of orthodox bands of groups .

Robert Horace Redfield, The generalized interval topology on distributive

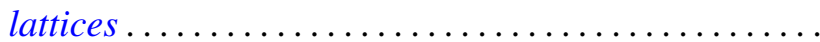

James Wilson Stepp, Algebraic maximal semilattices .... . .

Patrick Noble Stewart, A sheaf theoretic representation of rings with Boolean orthogonalities ........................

Ting-On To and Kai Wing Yip, A generalized Jensen's inequality......... 255

Arnold Lewis Villone, Second order differential operators with self-adjoint

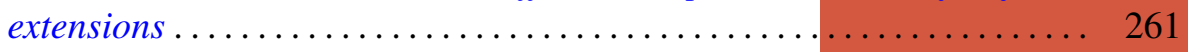

Martin E. Walter, On the structure of the Fourier-Stieltjes algebra ....... 267

John Wermer, Subharmonicity and hulls .................... 283

Edythe Parker Woodruff, A map of $E^{3}$ onto $E^{3}$ taking no disk onto a

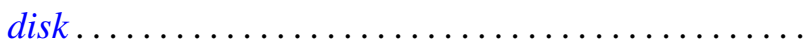

